\title{
Farsighted Stable Sets in Hotelling's Location Games
}

\author{
Junnosuke Shino*
}

\begin{abstract}
We apply farsighted stable set to two versions of Hotelling's location games: one with linear market and another with circular market. It is shown that there always exists a farsighted stable set in both games. In particular, the set of all location profiles that yields equal payoff across all players is shown to be a farsighted stable set. This stable set contains location profiles that reflect minimum differentiation as well as those profiles that reflect local monopoly. These results are in contrast to those obtained by equilibrium analysis. While the stable set uniquely exists when the number of players is 2 , the uniqueness is not guaranteed when $n \geq 3$. In particular, we exhibit multiple stable sets in three person location games. We provide possible interpretations of these farsighted stable sets from the viewpoint of players' bargaining power and coalition formation.
\end{abstract}

(Journal of Economic Literature Classification Number: C71, C72, L13)

Keywords: Farsighted stable set, Indirect dominance, Hotelling location game, Strategic form game with no-Nash equilibrium, Coalition formation

\section{Introduction}

Location model is one of the classical topics in the theory of product differentiation. To describe "whether sellers will tend to concentrate at one point or to disperse over the area" (Chamberlin (1933)) as an equilibrium is a fundamental question in this theory. In Hotelling's (1929) duopoly model with linear market, two firms are located at the centre of the market (Minimum Differentiation, hereafter $M D$ ) in the

${ }^{*}$ Department of Economics, Rutgers, The State University of New Jersey, NJ, USA. E-mail: jshino@econ.rutgers.edu 
unique equilibrium, rather than differentiating their location point (Local Monopoly, hereafter $L M)$. On the other hand, Eaton and R.G. Lipsey(1975) showed that when the number of firms is more than 2, or when the shape of the market is circle, MD does not necessarily arise in an equilibrium.

Furthermore, in the process of revealing these properties of equilibrium in spatial competition, another fundamental question emerged: As Eaton's paper noted, when the market is linear and the number of firms is three, there exists no pure strategy equilibrium. The existence of pure strategy equilibrium is recovered in a three person location game where after firms' location decisions, consumers has opportunity to relocate (see M. Fujita and J.F. Thisse (1986)). The location game is the special case of games with discontinuous payoff, for which did P. Dasgupta and E. Maskin (1986) show the existence of mixed strategy equilibrium.

In this paper, we employ stable set as a solution concept to analyze these issues in the location games. Specifically, we examine the farsighted stable set, due to Chwe (1994). Stable set was originally proposed by von Neumann and Morgenstern (1953) as a solution to cooperative games. The stable set is a set of outcomes satisfying internal stability and external stability. Internal stability requires that no outcome in the set dominates another. External stability requires that any outcomes outside the set must be dominated by an outcome in the set. In contrast to equilibrium concept, a deviation from an outcome in a stable set is deterred because such deviation induce further deviations, returning to an another outcome in the set by external stability. Internal stability ensures that a final outcome is not preferable for the deviation.

The farsighted stable set is also defined as a set of outcomes satisfying internal and external stabilities. The idea is that given an outcome as a status quo, "farsighted" players take individual or coalitional moves from it, which in turn may lead to further deviation by another coalition, and so on. Thus, it takes into account a domination of one outcome over an another via chain of such coalitional moves rather than myopic views in the original stable set concept ${ }^{1}$ (See Chwe(1994) and Greenberg(1990)).

In this paper, we consider two versions of location game: one with linear market and another with circular market, and we will see that farsighted stable set gives new points of view to the issues discussed above. First, as for the existence of a

\footnotetext{
${ }^{1}$ For a recent application of farsighted stable set to strategic form games, see Suzuki and Muto (2005). Properties of farsighted stable sets in cooperative game context are investigated in Beal et al. (2008).
} 
solution, it is shown that there always exists a farsighted stable set in both games, no matter what the number of player is. In particular, the set of all location profiles that yields equal payoff across all players is shown to be a farsighted stable set. Therefore, as for the possibility of description of $M D$ or $L M$, this stable set contains location profiles that reflect minimum differentiation as well as those profiles that reflect local monopoly. These results are in contrast to those obtained in analyses mentioned above. While the stable set uniquely exists when the number of players is 2 , the uniqueness is not guaranteed when $n \geq 3$. In particular, we exhibit multiple stable sets in three person location games. We provide possible interpretations of these farsighted stable sets from the viewpoint of players' bargaining power and coalition formation.

The organization of the rest of this paper is as follows. In Section 2, we give formal definition of farsighted stable set and brief comments on its interpretation. In Section 3, we describe two versions of location games as strategic form games. Main results are derived in Section 4. Some concluding remarks are made in Section 5 .

\section{Farsighted Stable Set}

In this section, we give formal definition of farsighted stable set in strategic form games and brief comments on its interpretation.

Let $G=\left(N,\left\{Z_{i}\right\}_{i \in N},\left\{u_{i}\right\}_{i \in N}\right)$ be a strategic form game where $N=\{1,2, \ldots, n\}$ is the set of players, $Z_{i}$ is the set of strategies of player $i$, and $u_{i}$ is the player $i$ 's utility function where $u_{i}: Z=\times_{i \in N} Z_{i} \longrightarrow \mathbb{R}$. We call $z=\left(z_{1}, \ldots, z_{n}\right) \in Z$ an outcome. When $\sum_{i \in N} u_{i}(z)=c$ for all $z \in Z$ and for some $c \in \mathbb{R}$, we call that $G$ is constant-sum.

A subset of players $S \subseteq N$ is called a coalition. For any two outcomes $v=$ $\left(v_{1}, v_{2}, \ldots, v_{n}\right) \in Z$ and $w=\left(w_{1}, w_{2}, \ldots, w_{n}\right) \in Z$, we say $w$ is induced from $v$ via coalition $S \subseteq N$, or, $S$ deviates from $v$ to $w$, denoted $v \longrightarrow_{S} w$, if $v_{i}=w_{i}$ for all $i \in N \backslash S . v \longrightarrow S w$ means that coalition $\mathrm{S}$ can move from $v$ to $w$ by itself.

For any two outcomes $v$ and $w$, we say $w$ indirectly dominates $v$, denoted $v \ll w$, if there exists a sequence of outcomes $\left(w^{0}, w^{1}, \ldots, w^{K}\right)$ with $v=w^{0}$ and $w=w^{K}$, and there exists a sequence of coalitions $\left(S_{0}, S_{1}, \ldots, S_{K-1}\right)$ such that for all $k \in$ $\{0,1, \ldots, K-1\}, w^{k} \longrightarrow S_{k} w^{k+1}$ and $u_{i}\left(w^{k}\right)<u_{i}\left(w^{K}\right)$ for all $i \in S_{k}$.

The situation behind the definition of indirect dominance is that any coalitions 
can be formed without binding contracts. For example, any members in the coalition $S_{k}$ can enter another coalition $S_{k+1}$, which induces further deviation. Moreover, it should be noted that players are assumed to be farsighted in the definition of indirect dominance. All members in $S_{k}$ compare current outcome $w^{k}$ with not immediate outcome $w^{k+1}$ but with possible final outcome $w^{K}$. Therefore, it is likely that $S_{k}$ moves from $w^{k}$ to $w^{k+1}$, which may not be prefered to $w^{k}$, anticipating this deviation induces further deviations.

When $K=1$, we call $w$ directly dominates $v$. Direct dominance relation between two outcomes was originally proposed by von Neunmann and Morgenstern(1953) in cooperative game context. Greenberg(1990) also introduced notion of direct dominance into noncooperative games. After that, however, direct dominance is criticized for representing only myopic players, and Harsanyi(1974) and Chwe(1994) proposed indirect dominance relation so as to be consistent with a farsighted player's behavior. As for more detailed discussion, see these articles.

Farsighted stable set is a solution concept based on the indirect dominance. A set of outcomes $V \subseteq Z$ is a farsighted stable set if (1)for all $v, w \in V$, neither $v \ll w$ nor $w \ll v$, and (2)for all $v \in Z \backslash V$, there exists $w \in V$ such that $v \ll w$. Conditions (1) and (2) are called internal stability and external stability, respectively.

A farsighted stable set can be interpreted as a set of outcomes which are supported by a "stable standard of behavior". Now suppose that players engage in open bargaining with moves and counter moves, given an outcome $x \in Z$ as a status quo. If we assume that players behave consistent with indirect dominance, it is natural to think that the set of all possible outcomes is $P(x)=\{x\} \cup\{y \in Z \mid x \ll z\}$. Furthermore, if players have common understanding about which outcomes are not excluded as a consequence of the open bargaining, we can define a mapping $\phi(\cdot)$ that assigns a subset of $P(x)$ as a set of possible agreement points, to each status quo $x$. In accordance with Greenberg(1990), we call $\phi$ a standard of behavior. In principle, $\phi$ can be any arbitrary mapping, but now consider the following stability condition on $\phi$;

$$
y \in \phi(x) \Longleftrightarrow y \nprec_{S} w \text { for all } w \in \phi(z) \text { such that } y \longrightarrow_{S} z^{2} .
$$

where $y \nprec_{S} w$ means that there exists $i \in S$ such that $u_{i}(y) \geq u_{i}(z)$. Then it can be shown that a farsighted stable set $V$ is identical to the set $\cup_{x \in Z} \phi(x)$ (See

\footnotetext{
${ }^{2}$ Note that $\phi$ does not necessarily uniquely exist.
} 
Xue(1998)), that is, a farsighted stable set can be interpreted as a set of outcomes which are supported by a certain "stable standard of behavior 3 ".

\section{3 n-Person Location Games}

In this section, we describe two versions of spatial competitions as a strategic form game: one with linear market and another with circular market. We call each game a linear location game and a circular location game, respectively.

In the linear location game, we assume that there is a "linear city," which is expressed as real number line $[0,1]$. $n$ firms produce identical physical good, and each of them chooses its location point on the line. A firm's production and location accompany no cost. Consumers are uniformly distributed on the line, and each of them always buys one unit of good from a firm which is nearest to her. A firm's utility is the share to the total market. Note that a firm's utility level for a certain location profile depends not only its own location point but also other ones. If multiple firms locate at the same point, we assume that they equally divide their share.

Denote linear location game by $G_{l}$. The set of players is $N=\{1, \ldots, n\}$, and player $i$ 's strategy set is $Z_{i}=[0,1]$. To describe $i$ 's utility function formally, as well as to make our analysis mathematically concise, we introduce some notations. First, for a outcome $z=\left(z_{1}, \ldots, z_{n}\right) \in Z$, let $k(z)$ be the number of distinct values of $\left(z_{1}, \ldots, z_{n}\right)$, and denote them, in an increasing order, $L_{1}(z), L_{2}(z), \ldots, L_{k(z)}(z)$. Denote a vector of location points by $L(z)=\left(L_{1}(z), L_{2}(z), \ldots, L_{k(z)}(z)\right)$. Note that $L(z)$ has finite elements since $k(z) \leq n$. Furthermore, for $L_{j}(z)(j \in\{1, \ldots, k(z)\})$, we denote the number of players locating at $L_{j}(z)$ by $F_{j}(z)$, and define $F(z)=$ $\left(F_{1}(z), F_{2}(z), \ldots, F_{k(z)}(z)\right)$. Finally, we denote the distance between $z_{i}$ and $z_{j}$ by $\left\|z_{i}-z_{j}\right\|=\left|z_{i}-z_{j}\right|$ where $|\cdot|$ denote absolute value.

We give an example in Figure 1 (left). In this example, $z=(3 / 5,1 / 4,0,1 / 4)$, $k(z)=3, L(z)=(0,1 / 4,3 / 5), F(z)=(1,2,1)$, and $\left\|z_{1}-z_{2}\right\|=7 / 20,\left\|z_{2}-z_{4}\right\|=0$, and so forth. $i$ 's utility is defined based on our assumption discussed above. For instance, the sum of player 2 and 4's share to the total market is $3 / 10$. Since they divide this equally, $u_{2}(z)=u_{4}(z)=3 / 20$. We can also check easily that

\footnotetext{
${ }^{3}$ More precisely, this standard of behavior is defined as optimistic standard of behavior, which was originally proposed in Chwe (1994). He also defined conservative standard of behavior and consistent set which is a set of outcome associated with this standard of behavior.
} 
$u_{1}(z)=23 / 40$ and $u_{3}(z)=1 / 8$. Note that $G_{l}$ is constant-sum.
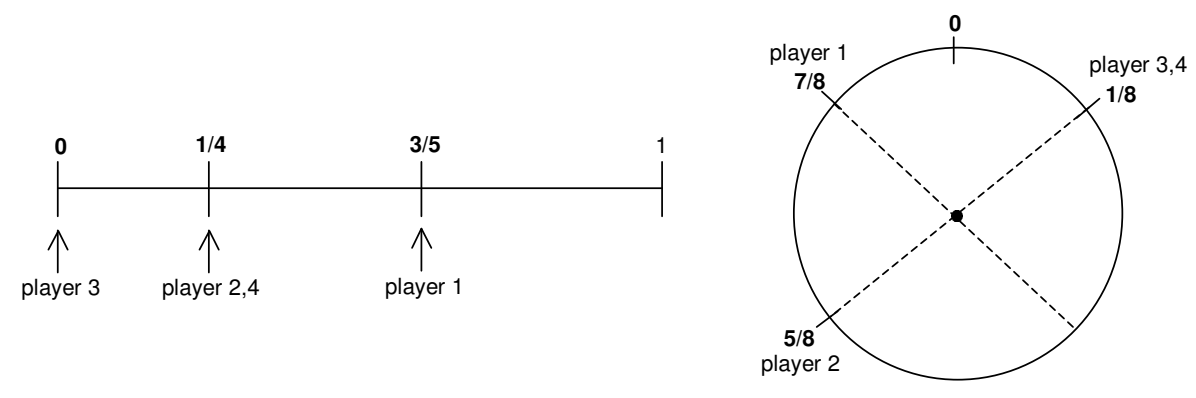

Figure 1: Linear Location Game (Left) and Circular Location Game (Right)

Formally, we define player $i$ 's utility function in $G_{l}$ as follows;

(1) When $k(z)=1$,

$$
u_{i}(z)=1 / n \quad \text { for all } i \in N
$$

(2) When $k(z)=2$,

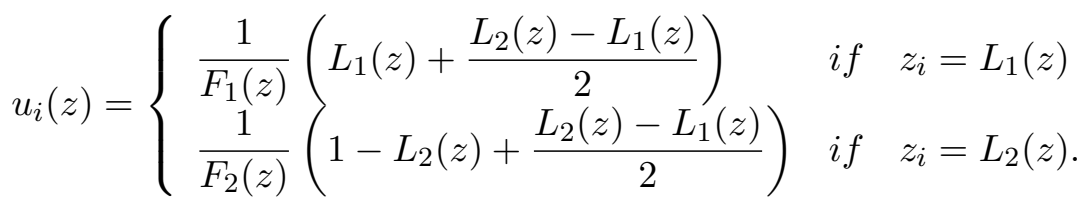

(3) When $k(z) \geq 3$,

$u_{i}(z)= \begin{cases}\frac{1}{F_{1}(z)}\left(L_{1}(z)+\frac{L_{2}(z)-L_{1}(z)}{2}\right) & \text { if } \quad z_{i}=L_{1}(z) \\ \frac{1}{F_{m}(z)}\left(\frac{L_{m+1}(z)-L_{m-1}(z)}{2}\right) & \text { if } \quad z_{i}=L_{m}(z)(m \neq 1, m \neq k(z)) \\ \frac{1}{F_{k(z)}(z)}\left(1-L_{k(z)}(z)+\frac{L_{k(z)}(z)-L_{k(z)-1}(z)}{2}\right) & \text { if } \quad z_{i}=L_{k(z)}(z) .\end{cases}$

In the circular location game, the only difference is that we assume a "circular city," which is expressed as a circle with a perimeter 1, instead of a linear city. Consumers are uniformly distributed on the perimeter, and each firm chooses its 
location point to maximize the share to the total market. We give a number 0 to an arbitrary point on the perimeter.

Denote the circular location game by $G_{c}$. Note that in $G_{c}$, player $i$ 's strategy set is $Z_{i}=[0,1)$. Furthermore, note that $k(z), L(z)$, and $F(z)$ can be defined in the same fashion as those in $G_{l}$. We define $\left\|z_{i}-z_{j}\right\|$ as a shorter length among two arcs formed by $z_{i}$ an $z_{j}$. An example is shown in Figure 1 (right). $z=(7 / 8,5 / 8,1 / 8,1 / 8)$, $k(z)=3, L(z)=(1 / 8,5 / 8,7 / 8), F(z)=(2,1,1)$, and $\left\|z_{1}-z_{2}\right\|=1 / 4,\left\|z_{1}-z_{3}\right\|=$ $1 / 4$, and so on. $i$ 's utility is also defined in the same manner as $G_{l}: u_{1}(z)=1 / 4$, $u_{2}(z)=3 / 8$, and $u_{3}(z)=u_{4}(z)=3 / 16$. Again, note that $G_{c}$ is constant-sum.

The formal expression of player $i$ 's utility function in $G_{c}$ can be expressed as follows;

(1) When $k(z)=1$,

$$
u_{i}(z)=1 / n \quad \text { for all } i \in N
$$

(2) When $k(z)=2$,

$$
u_{i}(z)=\frac{1}{2 F_{m}(z)} \quad \text { if } z_{i}=L_{m}(z) \quad(m=1,2)
$$

(3) When $k(z) \geq 3$,

$$
u_{i}(z)= \begin{cases}\frac{1}{F_{1}(z)}\left(\frac{1-L_{k(z)}(z)+L_{2}(z)}{2}\right) & \text { if } \quad z_{i}=L_{1}(z) \\ \frac{1}{F_{m}(z)}\left(\frac{L_{m+1}(z)-L_{m-1}(z)}{2}\right) & \text { if } \quad z_{i}=L_{m}(z)(m \neq 1, m \neq k(z)) \\ \frac{1}{F_{k(z)}(z)}\left(\frac{1-L_{k(z)-1}(z)+L_{1}(z)}{2}\right) & \text { if } \quad z_{i}=L_{k(z)}(z) .\end{cases}
$$

\section{Farsighted Stable Sets in Location Games}

In this section, we derive farsighted stable sets in each location game, $G_{l}$ and $G_{c}$. Before doing so, however, it is useful to mention some properties of indirect dominance relations and farsighted stable sets in general strategic form games.

\section{Remark 4.1}

In a strategic form game $G$, for all $x, y, z \in Z$, if $y \ll z, x \rightarrow_{S} y$ and $u_{i}(x)<u_{i}(z)$ for all $i \in S$, then $x \ll z$. 
Remark 4.1 is straightforward from the definition of the indirect dominance.

\section{Remark 4.2}

Let $G$ be a strategic form game and $V$ and $\tilde{V}$ be two different farsighted stable set. Then neither $\tilde{V} \subsetneq V$ nor $V \subsetneq \tilde{V}$ can be true.

Proof. ${ }^{4}$ Suppose that $\tilde{V} \subsetneq V$ and pick an outcome $x$ such that $x \in V$ and $x \notin \tilde{V}$. From the external stability of $\tilde{V}$, there exists $\tilde{x} \in \tilde{V}$ such that $x \ll \tilde{x}$. However, since $x \in V$ and $\tilde{x} \in V$, it contradicts the internal stability of $V$. Q.E.D.

\section{Lemma 4.1}

Let $G$ be a two-person constant-sum strategic form game and $V$ be a farsighted stable set in $G$. Then

$$
u_{i}(x) \geq \max _{z_{i} \in Z_{i}} \inf _{z_{j} \in Z_{j}} u_{i}(z) \text { for all } x \in V \text { and for all } i \in N \text {. }
$$

Proof. See Appendix.

Lemma 4.1 says that in any stable sets, every outcome in the set assigns every player equal to or greater than their "maxinf "value when the number of players is 2 and the game is constant. Note that $\min _{z_{j} \in Z_{j}} u_{i}(z)$ is not well-defined since $u_{i}(z)$ is not continuous. Thus, we consider "maxinf "value instead of "maxmin."

\subsection{Linear Location Game}

Now we derive farsighted stable sets in the linear and circular location games defined in the previous section. First, we examine farsighted stable sets in $G_{l}$.

\section{Theorem 4.1}

Let

$$
V_{e}=\left\{z \in Z \mid u_{i}(z)=1 / n \text { for all } i \in N\right\}
$$

then $V_{e}$ is a farsighted stable set in the linear location game $G_{l}$.

Proof. The internal stability of $V_{e}$ is obviously satisfied from the property of $V_{e}$. To check the external stability, pick $x^{0} \notin V_{e}$. Note that $k\left(x^{0}\right) \geq 2$ (otherwise $x^{0} \in V_{e}$ ) and we show this statement by using induction on $k\left(x^{0}\right)$.

\footnotetext{
${ }^{4}$ The proof is basically identical as seen in basic game theory textbooks. See M.Osbone and A.Rubinstein(1994), for instance.
} 
First, we show the following;

for every $x^{0} \notin V_{e}$ with $k\left(x^{0}\right)=2$, there exists $z \in V_{e}$ such that $x^{0} \ll z$.

Since $x^{0} \notin V_{e}$ and the game is constant-sum, there exists a location point $L_{m}\left(x^{0}\right)$ ( $m=1$ or 2 ) such that $u_{i}\left(x^{0}\right)<1 / n$ for all $i$ with $x_{i}^{0}=L_{m}\left(x^{0}\right)$. Let $S_{0}=\{i \in$ $\left.N \mid x_{i}^{0}=L_{m}\left(x^{0}\right)\right\}$ and consider $z \in Z$ such that (i) $z_{j}=x_{j}^{0}$ for all $j \in N \backslash S_{0}$ and (ii) $z_{i}=z_{j}$ for all $i \in S_{0}$. Note that $z \in V_{e}$ and the fact that $x^{0} \longrightarrow S_{0} z$ is possible and $u_{i}\left(x^{0}\right)<1 / n=u_{i}(z)$ for every $i \in S_{0}$ implies $x^{0} \ll z$.

Next, suppose that the following statement is true;

$$
\text { for every } \tilde{x}^{0} \notin V_{e} \text { with } k\left(\tilde{x}^{0}\right)=\tilde{k} \text {, there exists } z \in V_{e} \text { such that } \tilde{x}^{0} \ll z \text {, }
$$

and we must show;

for every $x^{0} \notin V_{e}$ with $k\left(x^{0}\right)=\tilde{k}+1$, there exists $z \in V_{e}$ such that $x^{0} \ll z$. (2)

Pick $x^{0} \notin V_{e}$ with $k\left(x^{0}\right)=\tilde{k}+1$. Similarly to the previous case, $x^{0} \notin V_{e}$ guarantee the existence of a location point $L_{m}\left(x^{0}\right)(m \in\{1, \ldots, \tilde{k}+1\})$ such that $u_{i}\left(x^{0}\right)<1 / n$ for all $i$ with $x_{i}^{0}=L_{m}\left(x^{0}\right)$. Let $S_{0}=\left\{i \in N \mid x_{i}^{0}=L_{m}\left(x^{0}\right)\right\}$ and consider $S_{0}$ 's deviation from $x^{0}$ to $\hat{x}^{0}$ such that (i) $\hat{x}_{j}^{0}=x_{j}^{0}$ for all $j \in N \backslash S_{0}$ and (ii) $\hat{x}_{i}^{0}=\hat{x}_{j}^{0}$ for all $i \in S_{0}$. If $\hat{x}^{0} \in V_{e}$, then $u_{i}\left(x^{0}\right)<1 / n=u_{i}\left(\hat{x^{0}}\right)$ thus $x^{0} \ll \hat{x}^{0}$. Therefore, (2) is true. If $\hat{x}^{0} \notin V_{e}$, then note that $k\left(\hat{x}^{0}\right)=\tilde{k}$ thus there exists $z \in V_{e}$ satisfying $\hat{x}^{0} \ll z$ from (1). Now Remark 4.1 is applied and we conclude that $x^{0} \ll z$. Q.E.D.

Theorem 4.1 says that a stable set $V_{e}$ always exists in $G_{l}$ whatever the number of player is. Note that $V_{e}$ contains all outcomes such that all players locate at a same point, each of those reflects $M D$. Moreover, $V_{e}$ also contains outcomes in which every player locates at different point and each of them enjoys its monopoly power in its own territory. Therefore, we can also see that certain outcomes in $V_{e}$ describe $L M$. Obviously, an outcome representing mixture of $M D$ and $L M$ is also in $V_{e}$.

Interestingly, the only required property for an outcome to be an element of $V_{e}$ is that the market share has to be divided equally among all players. One possible interpretation behind this farsighted stable set is that all players' bargaining powers are well-balanced thus they agree with a completely equal allocation. In this sense, $V_{e}$ can be interpreted as a consequence of grand coalition formation. We will discuss 
this point further in the case of $n=3$ after showing Remark 4.3.

Next, we examine some results about uniqueness.

First, we show that when the number of player is $2, V_{e}$ is the unique farsighted stable set in $G_{l}$

\section{Theorem 4.2}

When $n=2, V_{e}$ in Theorem 4.1 is the unique farsighted stable set in $G_{l}$.

Proof. Consider $G_{l}$ and suppose $n=2$. Note that $\max _{z_{i} \in Z_{i}} \inf _{z_{j} \in Z_{j}} u_{i}(z)=1 / 2$ for all $i \in\{1,2\}$ since

$$
\inf _{z_{j} \in Z_{j}} u_{i}\left(z_{i}, z_{j}\right)\left\{\begin{array}{l}
=\frac{1}{2} \text { when } \quad z_{i}=\frac{1}{2} \\
<\frac{1}{2} \text { when } \quad z_{i} \neq \frac{1}{2}
\end{array}\right.
$$

Therefore, from Lemma 4.1 and since the game is constant-sum, if $\mathrm{V}$ is a stable set in $G_{l}, u_{i}(x)=1 / 2$ for all $x \in V$ and for all $i \in\{1,2\}$. This means that if there exists a stable set $\tilde{V}$ with $\tilde{V} \neq V_{e}$, it has to satisfy $\tilde{V} \subsetneq V_{e}$ but it is impossible from Remark 4.2. Q.E.D.

When $n \geq 3$, the uniqueness of $V_{e}$ is not guaranteed. Remark 4.3 shows that when $n=3$, multiple stable sets exist in $G_{l}$

\section{Remark 4.3}

For $\alpha$ such that $\frac{1}{3}<\alpha<\frac{1}{2}$, let $i, j, k \in N$ and

$$
V_{i j}(\alpha)=\left\{z \in Z \mid u_{k}(z)=\alpha, u_{i}(z)=u_{j}(z)=\frac{1-\alpha}{2}\right\}
$$

then $V_{i j}(\alpha)$ is a farsighted stable set in $G_{l}$ when $n=3$.

Proof. In the following proof, we show $V_{23}(\alpha)$ is a farsighted stable set. The internal stability of $V_{23}(\alpha)$ is obviously satisfied. Before checking $V_{23}(\alpha)$ 's external stability, note that:

For $1 / 3<\alpha<1 / 2$, let $z^{L}$ and $z^{R}$;

$$
z^{L}=\left(z_{1}^{L}, z_{2}^{L}, z_{3}^{L}\right)=\left(2 \alpha-\frac{1}{2}, \frac{1}{2}, \frac{1}{2}\right) \quad z^{R}=\left(z_{1}^{R}, z_{2}^{R}, z_{3}^{R}\right)=\left(\frac{3}{2}-2 \alpha, \frac{1}{2}, \frac{1}{2}\right)
$$

then $z^{L}, z^{R} \in V_{23}(\alpha)$. Note that $1 / 6<z_{1}^{L}<1 / 2$ and $1 / 2<z_{1}^{R}<5 / 6$. 
Now we check the external stability of $V_{23}(\alpha)$. Pick $x \notin V_{23}(\alpha)$ and we will show that there exists $z \in V_{23}(\alpha)$ such that $x \ll z$.

First, suppose $k(x)=1$. (1)when $x_{1}=x_{2}=x_{3}=1 / 2$, player 1 deviates from $x$ to $z=z^{L}$ or $z=z^{R}$. Note that $z \in V_{23}(\alpha)$. Since $u_{1}(z)=\alpha>1 / 3, x \ll z$. (2)when $x_{1}=x_{2}=x_{3} \neq 1 / 2$, w.l.o.g, suppose that $x_{1}=x_{2}=x_{3}=1 / 2+d$ where $0<d \leq 1 / 2$. Assume that player 1 deviates from $x$ to $y=\left(z_{1}^{L}, x_{2}, x_{3}\right)$, then coalition $\{23\}$ deviates from $y$ to $z=z^{L}$. Note that $u_{2}(y)=u_{3}(y)=1 / 2\{(1 / 2-d)+1 / 2(d+$ $1 / 2-2 \alpha+1 / 2)\}=1 / 2(1-\alpha)-(1 / 4) d<u_{2}(z)=u_{3}(z)$ and $u_{1}(x)=1 / 3<u_{1}(z)$. Therefore, from the definition of indirect dominance relation, we conclude $x \ll z$.

Next, Suppose $k(x)=2$. We divide this case into the following three cases: (A), (B), and (C).

(A)Suppose $x_{1} \neq x_{2}=x_{3}$. (1)When $u_{1}(x)<\alpha$, consider player 1's deviation from $x$ to $y=\left(x_{2}, x_{2}, x_{3}\right)$. Since $k(y)=1$, there exists $z \in V_{23}(\alpha)$ such that $y \ll z$. Furthermore, since $u_{1}(x)<\alpha=u_{1}(z)$, Remark 4.1 implies that $x \ll z$. (2)Suppose $u_{1}(x)>\alpha$. Since $G_{l}$ is constant-sum, this is equivalent to $u_{2}(x)=u_{3}(x)<(1-\alpha) / 2$. Assume that coalition $\{23\}$ deviates from $x$ to $y=\left(x_{1}, x_{1}, x_{1}\right)$. Again, since $k(y)=$ 1 , there exists $z \in V_{23}(\alpha)$ such that $y \ll z$ and $u_{2}(x)=u_{3}(x)<u_{2}(z)=u_{3}(z)$, thus $x \ll z$. (3)Note that we don't need to consider the case $u_{1}(x)=\alpha$ because such $x$ is in $V_{23}(\alpha)$ from the assumption $x_{1} \neq x_{2}=x_{3}$.

(B)Suppose $x_{2} \neq x_{1}=x_{3}$. (1)when $u_{2}(x)<(1-\alpha) / 2$, consider player 2's deviation from $x$ to $y=\left(x_{1}, x_{1}, x_{3}\right)$ where $k(y)=1$. Same argument holds and it follows that there exists $z \in V_{23}(\alpha)$ such that $x \ll z$. (2)Suppose $u_{2}(x) \geq(1-\alpha) / 2$, which is equivalent to $u_{1}(x)=u_{3}(x) \leq(1+\alpha) / 4$. Note that $(1-\alpha) / 2<(1+\alpha) / 4$ from the assumption on $\alpha$. If $u_{1}(x)=u_{3}(x)<(1-\alpha) / 2$, then consider deviation of the coalition $\{13\}$ from $x$ to $y=\left(x_{2}, x_{2}, x_{2}\right)$ and note that there exists $z \in V_{23}(\alpha)$ such that $y \ll z$. Since $u_{1}(x)<(1-\alpha) / 2<\alpha=u_{1}(z)$ and $u_{3}(x)<(1-\alpha) / 2=$ $u_{3}(z)$, it follows that $x \ll z$. If $(1-\alpha) / 2 \leq u_{1}(x)=u_{3}(x) \leq(1+\alpha) / 4$, note that this is equivalent to $(1-\alpha) / 2 \leq u_{2}(x) \leq \alpha$. Now consider player 1's deviation from $x$ to $y=\left(x_{2}, x_{2}, x_{3}\right)$ and the coalition $\{12\}$ 's consecutive deviation from $y$ to $w=\left(x_{3}, x_{3}, x_{3}\right)$. Note that there exists $z \in V_{23}(\alpha)$ such that $w \ll z$. Now we need to check whether the sequence of the outcomes $(x, y, w, \ldots, z)$ satisfies the condition on $x \ll z$. First, $u_{1}(x) \leq(1+\alpha) / 4<\alpha=u_{1}(z)$ since $\alpha>1 / 3$. Next, since $L(x)=$ $L(y), u_{1}(y)=u_{2}(y)=u_{2}(x) / 2$, which implies that $(1-\alpha) / 4 \leq u_{1}(y)=u_{2}(y) \leq \alpha / 2$. Therefore, $u_{1}(y) \leq \alpha / 2<\alpha=u_{1}(z)$ and $u_{2}(y) \leq \alpha / 2<(1-\alpha) / 2=u_{2}(z)$ since $\alpha<1 / 2$. Thus, from Remark 4.1., we conclude that $x \ll z$. 
(C)When $x_{3} \neq x_{1}=x_{2}$, the proof is identical to the case (B).

Finally, suppose that Suppose $k(x)=3$. Since $x \notin V_{23}(\alpha)$ and $G_{l}$ is constantsum, there exists $i \in N$ such that $u_{i}(x)<u_{i}(\tilde{z})$ where $\tilde{z} \in V_{23}(\alpha)$. Take $i$ 's deviation from $x$ to $y$ satisfying $k(y)=2$, and note that we can always take such deviation. From the previous part, we know that there exists $z \in V_{23}(\alpha)$ such that $y \ll z$. From Remark 4.1, $x \ll z$. Q.E.D.

Now compare $V_{e}$ in Theorem 4.1 with $V_{i j}(\alpha)$ in Remark 4.3. First of all, note that $i$ 's "minsup "value in $G_{l}$ is $1 / 4$ when $n=3^{5}$. Minsup value is $i$ 's payoff which can be obtained against the other players' worst joint actions for him, provided that $i$ takes a best action to it. Thus, minsup value can be regarded as $i$ 's maximum payoff which can be obtained by his own, when the "worst scenario "for him happens. In $V_{e}$, every player gets the market share strictly greater than its minsup payoff $(1 / 4<1 / 3)$ and completely equal allocations are attained. Therefore, one possible interpretation of $V_{e}$ is that players form grand coalition for the purpose to avoid realizing their "worst scenario ".

On the other hand, note that in $V_{i j}(\alpha), 1 / 3<u_{k}(z)<1 / 2,1 / 4<u_{i}(z)<1 / 3$ and $1 / 4<u_{j}(z)<1 / 3$ for every $z \in V_{i j}(\alpha)$. Therefore, in $V_{i j}(\alpha)$, all players get market shares strictly greater than their minsup value. This is a common property we can observe both in $V_{e}$ and $V_{i j}(\alpha)$. However, at the same time, note that player $k$ succeeds to get the share strictly greater than that in $V_{e}$, while $i$ and $j$ 's share is strictly smaller than those in $V_{e}$. One possible interpretation of $V_{i j}(\alpha)$ is that these uneven allocations reflect each player's bargaining power. Namely, in $V_{i j}(\alpha)$, player $k$ has "strong" power relative to $i$ and $j$, while in $V_{e}$, each player's power is well-balanced. Coalition $\{i j\}$ convinces player $k$ to accept $V_{i j}(\alpha)$ by giving strictly greater payoff than what $k$ would get in $V_{e}$, and both of the "weaker"players in the coalition succeed to avoid falling into their minsup values.

\footnotetext{
${ }^{5}$ This can be checked as follows: (1)for any pairs $\left(x_{j}, x_{k}\right)$ satisfying $x_{j}=x_{k}$, player $i$ can get at least nearly to $1 / 2$ by locating close to $x_{j}=x_{k}$ but in the longer halfline formed by $x_{j}=x_{k}$ and the edge of the market. Then w.l.o.g, suppose $x_{j}<x_{k}$. (2)when $\left\|x_{j}-x_{k}\right\|>1 / 2, i$ locates in the open set $\left(x_{j}, x_{k}\right)$ and gets strictly more than $1 / 4$. (3)when $\left\|x_{j}-x_{k}\right\| \leq 1 / 2$ and $\left[x_{j}>1 / 4\right.$ or $\left.x_{k}<3 / 4\right], i$ gets at least $1 / 4$ by locating close to $x_{j}$ (or $x_{k}$ ) but in the halfline between 0 (or 1 ) and $x_{j}$ (or $x_{k}$ ). (4)Finally, suppose $\left\|x_{j}-x_{k}\right\| \leq 1 / 2$ and $\left[x_{j} \leq 1 / 4\right.$ and $\left.x_{k} \geq 3 / 4\right]$. In this case, the only possibility is $\left(x_{j}, x_{k}\right)=(1 / 4,3 / 4)$. For this pair, $i$ 's best response is locating in the closed set $[1 / 4,3 / 4]$ and gets $1 / 4$.
} 


\subsection{Circular Location Game}

Next, we examine farsighted stable sets in $G_{c}$.

\section{Theorem 4.3}

Let

$$
V_{e}=\left\{z \in Z \mid u_{i}(z)=1 / n \text { for all } i \in N\right\},
$$

then $V_{e}$ is a farsighted stable set in the circular location game $G_{c}$.

Proof. The proof is identical to the case of linear location game.

As stated in Theorem 4.1, Theorem 4.3 also shows that $V_{e}$ always exists in $G_{c}$, and $V_{e}$ includes outcomes representing both $M D$ and $L M$. It should be noted that the characteristics of $V_{e}$ does not depend on the shapes of the markets, which is contrast to results obtained by equilibrium analysis (See Eaton and Lipsey (1975)). Obviously, we can adopt same interpretation of $V_{e}$ as discussed after Theorem 4.1 in terms of grand coalition formation.

Then, we show that when the number of player is $2, V_{e}$ is the unique farsighted stable set in $G_{c}$.

\section{Theorem 4.4}

When $n=2, V_{e}$ in Theorem 4.3 is the unique farsighted stable set in $G_{c}$.

Proof. Consider $G_{c}$ and suppose $n=2$. Note that, in this case, $V_{e}=Z$. Since $V_{e}$ is a farsighted stable set from Theorem 4.3, Remark 4.2 directly implies that $V_{e}$ is the unique farsighted stable set. Q.E.D.

Similarly to $G_{l}$, the uniqueness of $V_{e}$ is not guaranteed when $n \geq 3$. We exhibit multiple stable sets in the case of $n=3$ in Remark 4.4.

\section{Remark 4.4}

For $\alpha$ such that $0<\alpha<\frac{1}{4}$, let $i, j, k \in N$ and

$$
V_{i j}(\alpha)=\left\{z \in Z \mid u_{i}(z)=\frac{1}{4}+\alpha, u_{j}(z)=\frac{1}{2}-\alpha, u_{k}(z)=\frac{1}{4}\right\}
$$

then $V_{i j}(\alpha)$ is a farsighted stable set in $G_{c}$ when $n=3$. 
Proof. In the following proof, we show that $V_{23}(\alpha)$ is a farsighted stable set. The internal stability of $V_{23}(\alpha)$ is obviously satisfied. Before checking $V_{23}(\alpha)$ 's external stability, for $0<\alpha<1 / 4$ and for $\left(x_{1}, x_{2}, x_{3}\right) \in Z$, define a set $\tilde{Z}\left(\alpha, x_{1}\right)$ such that

$$
\tilde{Z}\left(\alpha, x_{1}\right)=\left\{\tilde{z} \in Z \mid \tilde{z}_{1}=x_{1},\left\|\tilde{z}_{1}-\tilde{z}_{2}\right\|=2 \alpha,\left\|\tilde{z}_{2}-\tilde{z}_{3}\right\|=1 / 2\right\}
$$

Note that $\tilde{Z}\left(\alpha, x_{1}\right)$ contains two elements and note that if $\tilde{z} \in \tilde{Z}\left(\alpha, x_{1}\right)$, then $\tilde{z} \in$ $V_{23}(\alpha)$. Now we check the external stability of $V_{23}(\alpha)$. Pick $x \notin V_{23}(\alpha)$ and we will show that there exists $z \in V_{23}(\alpha)$ such that $x \ll z$.

First, pick $x \notin V_{23}(\alpha)$ such that $k(x)=1$. Pick $\tilde{z} \in \tilde{Z}\left(\alpha, x_{1}\right)$ and note that from the assumption on $\alpha$, either $u_{2}(\tilde{z})>1 / 3$ or $u_{3}(\tilde{z})>1 / 3$ is satisfied. (1)Suppose $u_{2}(\tilde{z})>1 / 3$. Then take player 2 's deviation from $x$ to $y=\left(x_{1}, \tilde{z}_{2}, x_{3}\right)$ and player 3 's consecutive deviation from $y$ to $z=\tilde{z}$. Now we need to check whether the sequence of the outcomes $(x, y, z)$ satisfies the condition on $x \ll z$. First, $u_{2}(x)=1 / 3<u_{2}(z)$. Next, $u_{3}(y)=1 / 4<1 / 2-\alpha=u_{3}(z)$ since $0<\alpha<1 / 4$. Therefore, $x \ll z$ holds. (2)Suppose $u_{3}(\tilde{z})>1 / 3$. In this case, the sequence of deviations $x \longrightarrow_{\{3\}} y \longrightarrow_{\{2\}} z$ such that $y=\left(x_{1}, x_{2}, \tilde{z}_{3}\right)$ and $z=\tilde{z}$ satisfies the condition on $x \ll z$.

Next, suppose $k(x)=2$. We divide this case into the following three cases: (A), $(\mathrm{B})$, and $(\mathrm{C})$.

(A)Suppose $x_{1} \neq x_{2}=x_{3}$. Consider a deviation of the coalition $\{23\}$ from $x$ to $z$ where $z \in \tilde{Z}\left(\alpha, x_{1}\right)$. Since $u_{2}(x)=1 / 4<1 / 4+\alpha=u_{2}(z)$ and $u_{3}(x)=1 / 4<$ $1 / 2-\alpha=u_{3}(z), x \ll z$.

(B)Suppose $x_{2} \neq x_{1}=x_{3}$. Take player 3's deviation from $x$ to $y=\left(x_{1}, x_{2}, x_{2}\right)$ and coalition $\{23\}$ 's further deviation from $y$ to $z$ where $z \in \tilde{Z}\left(\alpha, x_{1}\right)$. Since $u_{3}(x)=$ $1 / 4<u_{3}(z), u_{2}(y)=1 / 4<u_{2}(z)$, and $u_{3}(y)=1 / 4<u_{3}(z)$, we conclude $x \ll z$.

(C)When $x_{3} \neq x_{1}=x_{2}$, take the sequence of deviations $x \longrightarrow\{2\} y \longrightarrow\{23\} z$ such that $y=\left(x_{1}, x_{3}, x_{3}\right)$ and $z \in \tilde{Z}\left(\alpha, x_{1}\right)$. Then as discussed in (B), $x \ll z$ holds.

Finally, suppose that Suppose $k(x)=3$. Since $x \notin V_{23}(\alpha)$ and $G_{c}$ is constantsum, there exists $i \in N$ such that $u_{i}(x)<u_{i}(\hat{z})$ where $\hat{z} \in V_{23}(\alpha)$. Take $i$ 's deviation from $x$ to $y$ where $k(y)=2$, and note that we can always take such deviation. From the previous part, we know that there exists $z \in V_{23}(\alpha)$ such that $y \ll z$. Thus from Remark 4.1, $x \ll z$. Q.E.D. 
Now compare $V_{e}$ in Theorem 4.3 with $V_{i j}(\alpha)$ in Remark 4.4. First, in $G_{c}$, minsup value is $1 / 4$ when $n=3{ }^{6}$, which is identical to that in $G_{l}$. Therefore, $V_{e}$ gives every player the market share strictly greater than its minsup payoff, and completely equal allocations are attained. Thus, we can interpret $V_{e}$ as a result of grand coalition formation, as discussed in linear market case.

On the other hand, note that in $V_{i j}(\alpha), 1 / 4<u_{i}(\cdot)<1 / 2$ and $1 / 4<u_{j}(\cdot)<1 / 2$. The common property of $V_{i j}(\alpha) \mathrm{s}$ in $G_{l}$ and $G_{c}$ is that every player gets more than his minsup value. One interesting point uniquely lies in $G_{c}$ comes from the fact that at least one of $u_{i}(\cdot)$ and $u_{j}(\cdot)$ is always strictly greater than $1 / 3$. Keep this in mind, we can interpret $V_{i j}(\alpha)$ in $G_{c}$ as follows. Player $i$ possessing relatively "strong" bargaining power proposes to form coalition $\{i j\}$ to player $j$, guaranteeing to give $j$ strictly greater share than $j$ 's minsup value. Which $\alpha$ is realized depends on relative strength of $j$ 's bargaining power over $i$. For example, when $j$ 's power is not so much weaker than that of $i, i$ and $j$ are likely to reach an agreement that both of them get share more than $1 / 3(1 / 12<\alpha<1 / 6$ would be realized). On the other hand, if $j$ 's bargaining power over $i$ is weak, it seems to be natural to predict that $j$ would be forced to agree with a share greater than minsup value but less than $1 / 3(\alpha>1 / 6)$, which would have been attained if their power were well balanced.

Recall that when considering $V_{i j}(\alpha)$ in $G_{l}, i$ and $j$ form coalition and both of them are assumed to have "weaker" bargaining power. In $G_{c}$, contrastingly, we can interpret that at least one of the members of the coalition can be assumed to have "strong" bargaining power in $V_{i j}(\alpha)$.

\section{Concluding Remarks}

In this paper we studied farsighted stable sets in location games when firms act with farsightedness. We showed that there always exists a farsighted stable set, which depicts both of two seemingly contrast phenomena; $M D$ and $L M$. These phenomena were integrated under the same cause; completely equal allocations between all players. Then we showed that the stable set exists uniquely when

\footnotetext{
${ }^{6}$ Check $i$ 's minsup value. First, if $x_{j}=x_{k} i$ 's best response is to locate any points different from $x_{j}=x_{k}$ and get $1 / 2$. Next, if $\left\|x_{j}-x_{k}\right\|=1 / 2, i$ gets $1 / 4$ no matter which point he chooses. Finally, if $x_{j} \neq x_{k}$ and $\left\|x_{j}-x_{k}\right\|<1 / 2, i$ can get the share strictly greater than $1 / 4$ by locating in the wider arc of the two formed by $x_{j}$ and $x_{k}$. Thus note that in this case, "minmax "value is well defined, instead of "minsup" one. But here we use the term "minsup "to keep consistency of our usage of the term.
} 
$n=2$, while other stable sets exist when $n=3$. In the case of $n=3$, we also provided a possible interpretation that all of these farsighted stable sets can be seen as a consequence of players' coalition formation.

We conclude this paper by pointing out several questions for further research. First, our approach in this paper reminds us of another notion of farsighted stability proposed by Chwe (1994), which is called consistent set. As discussed in his paper, consistent set can be regarded as representation of players' conservative stable standard of behavior, while farsighted stable set can be considered as optimistic one. By applying consistent set to location games, we might obtain some further insights.

Next, in this paper we did not find all farsighted stable sets in location games in the case of $n=3$. Moreover, when $n>3$, we did not show any statements about the uniqueness of $V_{e}$. Finding other stable sets in these cases could be an interesting topic.

Finally, one interesting question is which and how a certain farsighted stable set is realized corresponding each player's bargaining power. For example, as discussed in section 4 , we showed that $V_{e}$ and $V_{i j}(\alpha)$ reflect players' underling bargaining powers in each case. Moreover, in a specific $V_{i j}(\alpha)$, a realized $\alpha$ also depends on their bargaining powers. To answer which $\alpha$ is realized, which coalition formation are plausible for a certain stable set, and more generally, which stable standard of behavior would be formed among all players, we need to formulate the dynamic process of communication or bargaining with farsighted players.

\section{Appendix}

Proof of Lemma 4.1. Let $G$ be a two person constant-sum strategic form game and $V$ a stable set in $G$. Assume that, in negation, there exists $\tilde{z} \in V$ and there exists $i \in N$ such that

$$
u_{i}(\tilde{z})<\max _{z_{i} \in Z_{i}} \inf _{z_{j} \in Z_{j}} u_{i}(z),
$$

and we derive contradiction. Let $z_{i}^{*}$ be $i$ 's "maxinf" strategy, that is, $\inf _{z_{j} \in Z_{j}} u_{i}\left(z_{i}^{*}, z_{j}\right)=$ $\max _{z_{i} \in Z_{j}} \inf _{z_{j} \in Z_{j}} u_{i}\left(z_{i}, z_{j}\right)$. Then, from the assumption,

$$
u_{i}\left(\tilde{z}_{i}, \tilde{z}_{j}\right)<\max _{z_{i} \in Z_{i}} \inf _{z_{j} \in Z_{j}} u_{i}(z)=\inf _{z_{j} \in Z_{j}} u_{i}\left(z_{i}^{*}, z_{j}\right)
$$


and since $\inf _{z_{j} \in Z_{j}} u_{i}\left(z_{i}^{*}, z_{j}\right) \leq u_{i}\left(z_{i}^{*}, \tilde{z}_{j}\right)$,

$$
u_{i}\left(\tilde{z}_{i}, \tilde{z}_{j}\right)<u_{i}\left(z_{i}^{*}, \tilde{z}_{j}\right)
$$

Therefore, from the definition of indirect dominance relation, $\left(\tilde{z}_{i}, \tilde{z}_{j}\right) \ll\left(z_{i}^{*}, \tilde{z}_{j}\right)$. Since $\left(\tilde{z}_{i}, \tilde{z}_{j}\right) \in V,\left(z_{i}^{*}, \tilde{z}_{j}\right) \notin V$ must be true to preserve the internal stability of $V$. From the external stability, it follows that there has to exist $\left(\tilde{w}_{i}, \tilde{w}_{j}\right) \in V$ such that $\left(z_{i}^{*}, \tilde{z}_{j}\right) \ll\left(\tilde{w}_{i}, \tilde{w}_{j}\right)$. From the definition of indirect dominance, the following has to be satisfied;

there exists a sequence of outcomes $\left(w^{0}, w^{1}, \ldots, w^{K}\right)$ with $w^{0}=\left(z_{i}^{*}, \tilde{z}_{j}\right)$ and $w^{K}=\left(\tilde{w}_{i}, \tilde{w}_{j}\right)$, and there exists a sequence of coalitions $\left(S_{0}, S_{1}, \ldots, S_{K-1}\right)$ such that for all $k \in\{0,1, \ldots, K-1\},(A) w^{k} \longrightarrow S_{k} w^{k+1}$ and $(B) u_{i}\left(w^{k}\right)<u_{i}\left(w^{K}\right)$ for all $i \in S_{k}$.

Now consider a coalition $S_{0}$ in (4) above.

First, suppose $\underline{i \in S_{0}}$. Since $\left(z_{i}^{*}, \tilde{z}_{j}\right) \ll\left(\tilde{w}_{i}, \tilde{w}_{j}\right), u_{i}\left(z_{i}^{*}, \tilde{z}_{j}\right)<u_{i}\left(\tilde{w}_{i}, \tilde{w}_{j}\right)$. From the inequality (3) above, it follows that $u_{i}\left(\tilde{z}_{i}, \tilde{z}_{j}\right)<u_{i}\left(\tilde{w}_{i}, \tilde{w}_{j}\right)$. Since $\left(\tilde{z}_{i}, \tilde{z}_{j}\right) \longrightarrow{ }_{\{i\}}$ $\left(z_{i}^{*}, \tilde{z}_{j}\right)$ is possible, from Remark $4.1,\left(\tilde{z}_{i}, \tilde{z}_{j}\right) \ll\left(\tilde{w}_{i}, \tilde{w}_{j}\right)$. However, since $\left(\tilde{z}_{i}, \tilde{z}_{j}\right) \in V$ and $\left(\tilde{w}_{i}, \tilde{w}_{j}\right) \in V$, it contradicts the internal stability of $V$.

Next, suppose $S_{0}=\{j\}$. (A) if $S_{1}=\cdots=S_{K-1}=\{j\}$ in the sequence of coalition of $(4)$, note that $\left(\tilde{w}_{i}, \tilde{w}_{j}\right)=\left(z_{i}^{*}, \tilde{w}_{j}\right)$. In this case, $u_{i}\left(\tilde{z}_{i}, \tilde{z}_{j}\right)<\inf _{z_{j} \in Z_{j}} u_{i}\left(z_{i}^{*}, z_{j}\right) \leq$ $u_{i}\left(z_{i}^{*}, \tilde{w}_{j}\right)=u_{i}\left(\tilde{w}_{i}, \tilde{w}_{j}\right)$. Therefore, since $u_{i}\left(\tilde{z}_{i}, \tilde{z}_{j}\right)<u_{i}\left(\tilde{w}_{i}, \tilde{w}_{j}\right),\left(z_{i}^{*}, \tilde{z}_{j}\right) \ll\left(\tilde{w}_{i}, \tilde{w}_{j}\right)$ and $\left(\tilde{z}_{i}, \tilde{z}_{j}\right) \longrightarrow\{i\}\left(z_{i}^{*}, \tilde{z}_{j}\right)$ is possible, $\left(\tilde{z}_{i}, \tilde{z}_{j}\right) \ll\left(\tilde{w}_{i}, \tilde{w}_{j}\right)$, from Remark 4.1. Again, this violates the internal stability of $V$. (B)Suppose there exists a coalition $S_{p}$ $(p \in\{1, \ldots, K-1\})$ such that $i \in S_{p}$. Let $S_{\tilde{p}}$ be the first coalition including $i$ in the sequence of coalitions in (4), that is, if $\tilde{k}<\tilde{p}$, then $S_{\tilde{k}}=\{j\}$. Now consider $\left(w_{i}^{\tilde{p}}, w_{j}^{\tilde{p}}\right) \longrightarrow S_{\tilde{p}}\left(w_{i}^{\tilde{p}+1}, w_{j}^{\tilde{p}+1}\right)$ in (4). Since $z_{i}^{*}=w_{i}^{0}=w_{i}^{1}=\ldots=w_{i}^{\tilde{p}}$, this is equal to $\left(z_{i}^{*}, w_{j}^{\tilde{p}}\right) \longrightarrow S_{\tilde{p}}\left(w_{i}^{\tilde{p}+1}, w_{j}^{\tilde{p}+1}\right)$. Recall that $i \in S_{\tilde{p}}$ thus from the definition of indirect dominance, $u_{i}\left(z_{i}^{*}, w_{j}^{\tilde{p}}\right)<u_{i}\left(\tilde{w}_{i}, \tilde{w}_{j}\right)$. Furthermore, $u_{i}\left(\tilde{z}_{i}, \tilde{z}_{j}\right)<\inf _{z_{j} \in Z_{j}} u_{i}\left(z_{i}^{*}, z_{j}\right) \leq$ $u_{i}\left(z_{i}^{*}, \tilde{w}_{j}^{\tilde{p}}\right)$. Therefore, $u_{i}\left(\tilde{z}_{i}, \tilde{z}_{j}\right)<u_{i}\left(\tilde{w}_{i}, \tilde{w}_{j}\right)$. Now recall that $\left(\tilde{z}_{i}, \tilde{z}_{j}\right) \longrightarrow\{i\}\left(z_{i}^{*}, \tilde{z}_{j}\right)$ is possible and $\left(z_{i}^{*}, \tilde{z}_{j}\right) \ll\left(\tilde{w}_{i}, \tilde{w}_{j}\right)$. Thus, Remark 4.1 implies $\left(\tilde{z}_{i}, \tilde{z}_{j}\right) \ll\left(\tilde{w}_{i}, \tilde{w}_{j}\right)$, which violates the internal stability of $V$. Q.E.D. 


\section{References}

[1] Beal.S., Durieu.J., and Solal.P. (2008). Farsighted Coalitional Stability in TUGames. Mathematical Social Sciences. 56, 303-313.

[2] Chamberlin. (1933). The theory of Monopolistic Competition. Harvard University Press.

[3] M. S. Chwe. (1994). Farsighted Coalitional Stability, Jounal of Economic Theory 63, 299-325.

[4] Dasgupta, P., and E. Maskin (1986). The Existence of Equilibrium in Discontinuous Economic Games, II: Applications, Review of Economic Studies 53, 27-42.

[5] Eaton, B. C., and R. Lipsey (1975). The Principle of Minimum Differentiation Reconsidered: Some New Developments in the Theory of Spatial Competition, Review of Economic Studies 42, 27-49.

[6] M. Fujita and J.F. Thisse (1986). Spatial Competition with a Land Market; Hotelling and , Review of Economic Studies 42, 27-49.

[7] J. Greenberg. (1990). The Theory of Social Situations: An Alternative Game Theoretic Approach. Cambridge Univ. Press, Cambridge.

[8] J. Harsanyi. (1974). An Equilibrium-Point Interpretation of Stable Sets and a Proposed Alternative Definition., Management Sci 20, 1472-1495.

[9] H. Hotelling. (1929). The Stability of Competition., Economic journal 39, 41-57.

[10] J. Osborne. and A. Rubinstein. (1994). A Course In Game Theory. The MIT Press.

[11] A. Suzuki and S. Muto. (2005). Farsighted Stability in an n-Person Prisoner's Dilemma., International Journal of Game Theory 33, 431-445.

[12] J.von Neumann and O.Morgenstern. (1953). Theory of Games and Economic Behavior Third Edition. Princeton Univ. Press, Princeton.

[13] L. Xue. (1998). Calitional Stability under Perfect Foresight, Economic Theory 11, 603-627 